\title{
A method for multi-class sentiment classification based on an improved one-vs-one (OVO) strategy and the support vector machine (SVM) algorithm
}

\author{
Yang Liu ${ }^{\text {a }}$ Jian-Wu Bi ${ }^{\text {a }}$, Zhi-Ping Fan ${ }^{\text {a, b, * }}$
}

\begin{abstract}
Multi-class sentiment classification is a valuable research topic with extensive applications; however, studies in the field remain relatively scarce. In the present paper, a method for multiclass sentiment classification based on an improved one-vs-one (OVO) strategy and the support vector machine (SVM) algorithm is proposed. First, an improved OVO strategy is proposed wherein the relative competence weight of each binary classifier is determined according to the $K$ nearest neighbors and the class center of each class in the training sample set concerning the binary classifier. A method for multi-class sentiment classification is proposed based on this improved OVO strategy and the SVM algorithm. After converting the training texts into term feature vectors, the important features (terms) for multi-class sentiment classification are selected using the information gain (IG) algorithm. A binary SVM classifier is then trained on the training feature vectors of each pair of sentiment classes. To identify the sentiment class of a test text, a confidence score matrix of multiple SVM classifiers is constructed based on the results of multiple SVM classifiers. Using this score matrix, the sentiment class of the test text can be determined using the improved OVO strategy. The results of our experimental studies show that the performance of the proposed method is significantly better than that of the existing methods for multi-class sentiment classification.
\end{abstract}

Keywords: Multi-class sentiment classification; One-vs-one (OVO) strategy; Support vector machine (SVM) algorithm; Experimental study 


\section{Introduction}

With the rapid development of information technology, an increasingly greater number of people are surfing the web. There are more than 3 billion internet users worldwide, and the number of internet users continues to grow rapidly [19]. Internet users typically express their opinions on social issues and share their experiences about products or services by posting online texts to various websites $[32,48]$. These online texts are valuable for the improvement of government, company and consumer decision making. Governments can make sounder public decisions by analyzing their citizens' online texts on social issues [3], companies can identify product weaknesses and forecast market demand by analyzing online product reviews $[6,22,25]$ and consumers can make suitable purchasing decisions by identifying the sentiment orientation of a large number of online product reviews $[18,46]$. Several studies on opinion mining and sentiment analysis have been conducted to automatically mine the opinions embedded in the vast repository of online texts and analyze the sentiment classes of a massive number of online texts. In these studies, sentiment classification is regarded as an important research topic for identifying the sentiment classes of online texts $[6,18,22,25,46$, 48]. Over the past decade, dozens of studies have introduced methods or classifiers for sentiment classification. It is vital to mention that only two types of sentiment classes are considered in most of the existing studies, i.e., the sentiment class of each text is classified as having either positive or negative sentiment orientation $[2,28,32,34]$. The sole consideration of positive and negative sentiment classes or online text orientations is regarded as excessive minimalism $[34,39]$. Multiple sentiment classes of online texts would enable more highly accurate and effective analyses for supporting the decisions of governments [3], companies $[6,21,22,25]$ and consumers $[18,46]$. Thus, multi-class sentiment classification is a valuable research topic with extensive applications.

Several studies on multi-class sentiment classification can be found in the literature. Pang and Lee [29] proposed a meta-algorithm for multi-class sentiment classification based on metric labeling. In their study, two sentiment classification systems of movie reviews were constructed, and sentiment classes using 3-and 4-class sentiment classification were assigned to each movie review. The effectiveness of the authors' meta-algorithm for multi-class sentiment classification based on metric labeling was illustrated by comparing the algorithm 
with one-vs-all SVM classification and SVM regression. Considering that texts with labeled sentiment classes are relatively scarce, Goldberg and Zhu [15] proposed a graph-based semisupervised algorithm. In the algorithm, an undirected graph is constructed based on the labeled and unlabeled texts, and a smooth rating function is obtained by solving an optimization problem. The sentiment classes of unlabeled texts were determined as per the smooth rating function. The movie review dataset built by Pang and Lee [29] was used to illustrate the effectiveness of the proposed algorithm. Wilson et al. [44] conducted an experimental study on multi-class sentiment classification. In their study, text dependency trees were constructed using a linguistic analysis technique to reflect the grammatical structures of the texts. The effects of the dependency trees on multi-class sentiment classification were verified by combining them with different machine learning algorithms. The experimental results showed that dependency trees improved the performance of various classifiers for multi-class sentiment classification. Bickerstaffe and Zukerman [4] proposed a hierarchical classifier for multi-class sentiment classification. In their study, decision tree structures are first constructed by calculating inter-class similarities using a shortest-spanning algorithm, where each leaf represents a target sentiment class. Then, a hierarchical classifier is obtained by combining standard binary classifiers in accordance with the decision tree structures. Their experiments were conducted to verify the advantages of the proposed classifier over the multi-class sentiment classifiers proposed by Pang and Lee [29]. Cao and Zukerman [5] developed a probabilistic approach for multi-class sentiment classification by incorporating lexical information and specific grammatical structure characteristics into the Naïve Bayes (NB) classifier. Their approach performed at least as well as the method proposed by Bickerstaffe and Zukerman [4]. To classify the sentiment classes of short informal text, Thelwall et al. [40] proposed the SentiStrength algorithm. In their study, a sentiment word strength list is first determined using a machine learning algorithm, then the SentiStrength algorithm is developed by considering several characteristics of short informal text such as spelling correction, booster words, a negating word list, repeated letters, etc. The accuracies of the SentiStrength algorithm on identifying the sentiment classes of short informal texts with positive and negative sentiment orientations were $72.8 \%$ and $60.6 \%$, respectively. Thus, the SentiStrength algorithm does not perform as well on identifying the sentiment classes of short informal texts with negative sentiment orientation as those with 
positive sentiment orientation. To address this shortcoming, Thelwall et al. [41] proposed a revised algorithm called SentiStrength 2. In SentiStrength 2, the lexicon of negative terms is significantly extended by incorporating negative General Inquirer terms [36] into the original SentiStrength algorithm.

Previous studies on multi-class sentiment classification have made significant contributions to the field; however, they are limited to the aforementioned methods. In addition, the classification accuracy for multi-class sentiment classification is relatively low, which restricts the application of the existing methods to government [3], company $[6,22,25]$ and consumer support [18, 46]. Thus, more efficient methods for multi-class sentiment classification are required. In the existing studies on binary sentiment classification $[2,23,26$, 28], different machine learning algorithms are used to develop the methods for binary sentiment classification, in which the support vector machine (SVM) is regarded as one of the most effective machine learning algorithms for sentiment classification. Thus, the SVM algorithm has the potential to support increasingly efficient methods for multi-class sentiment classification. Because the SVM algorithm is a binary classifier, decomposition strategies must be used when the SVM algorithm is used for multi-class sentiment classification. The one-vs-one (OVO) strategy is regarded as one of the most effective decomposition strategies available [11]. Several OVO strategies have been proposed for combining the results of binary classifiers; however, the existing OVO strategies have certain limitations, which are illustrated in Section 2.1. Thus, to develop new methods for multi-class sentiment classification based on the SVM algorithm, an improved OVO strategy is proposed in the present study, followed by a novel method for multi-class sentiment classification using an improved OVO strategy and the SVM algorithm.

The remainder of this paper is arranged as follows: Section 2 proposes an improved OVO strategy, Section 3 presents the proposed method for multi-class sentiment classification, Section 4 describes the setup of the experimental framework and Section 5 presents experimental studies that illustrate the effectiveness of the proposed method. Finally, Section 6 summarizes and highlights the major contributions of this paper.

\section{The improved OVO strategy}

To develop a method for multi-class sentiment classification based on the SVM algorithm, 
an improved OVO strategy is proposed in this section. A brief introduction to the OVO strategy is given in Section 2.1. Next, the development of the improved OVO strategy is described in Section 2.2. Finally, an illustrative example is given in Section 2.3 to clearly illustrate the advantages of the improved OVO strategy.

\subsection{A brief introduction to the OVO strategy}

The OVO strategy is one of the most commonly used methods for transforming multi-class classification problems into multiple binary classification problems $[11,20]$. In the OVO strategy, an $m$-class classification problem is transformed into $m(m-1) / 2$ binary classification problems. To accomplish this, $m(m-1) / 2$ training sample sets are first constructed in accordance with the training samples pertaining to $m$ classes, where each training sample set is the union of the training samples for two classes. Then, a binary classifier is trained on each training sample set using a machining learning algorithm, i.e., $m(m-1) / 2$ binary classifiers can be obtained, which correspond to $m(m-1) / 2$ training

sample sets. To determine the class of a test sample, the obtained $m(m-1) / 2$ binary classifiers are used to respectively identify the class of the test sample, and the final class of the test sample is determined by integrating the results of the $m(m-1) / 2$ binary classifiers. A score matrix is typically required, i.e.,

$$
R=\left(r_{i i^{\prime}}\right)_{m \times m}=\left(\begin{array}{cccc}
- & r_{12} & ? & r_{1 m} \\
r_{21} & - & ? & r_{2 m} \\
? & ? & ? \\
r_{m 1} & r_{m 2} & ? & -
\end{array}\right)
$$

where $r_{i i^{\prime}} \in[0,1]$ is the confidence of the binary classifier for discriminating classes $i$ and $i^{\prime}$ in favor of class $i$. The confidence of the binary classifier in favor of class $i^{\prime}$ can be computed by $r_{i^{\prime} i}=1-r_{i i^{\prime}}$. The value of $r_{i i^{\prime}}$ is associated with the number of training samples and categories as well as the results of different classifiers. In the present study, the SVM algorithm is used to train our binary classifiers; the details for calculating the value of $r_{i i^{\prime}}$ are given in Section 3.3.2.

To determine the class of a test sample according to the score matrix $R=\left(r_{i i^{\prime}}\right)_{m \times m}$, several 
OVO strategies have been proposed, such as the voting OVO (V-OVO) strategy [12], the weighted voting OVO (WV-OVO) strategy [17], etc. A description of the existing OVO strategies is omitted in this paper for the sake of brevity. An extensive and detailed description of OVO strategies can be found in the literature [10]. However, most of the existing studies on OVO strategies virtually ignore the competence of different binary classifiers [9, 17]. Because a binary classifier is trained using the training sample set regarding two classes, the binary classifier is capable for classifying the test samples belonging to the two classes, but not those belonging to the other $m-2$ classes [11]. In other words, with respect to a given test sample, $m-1$ of $m(m-1) / 2$ binary classifiers are regarded as competent classifiers and the remaining $m(m-1) / 2-(m-1)$ binary classifiers are regarded as non-competent classifiers. For example, consider a test sample belonging to the ${ }^{i}$ th class, $i \in\{1,2,0, m\}$. The binary classifier trained on the training sample set regarding the $i$ th and $i^{\prime}$ th classes is a competent classifier for identifying the sentiment class of the test sample where $\left.i^{\prime}=1,2, ?\right], m$ and $i^{\prime} \neq i$. Thus, $m-1$ binary classifiers are regarded as competent classifiers because there are $m-1$ possible values of $i^{\prime}, i^{\prime}=1,2, ?, m$ and $i^{\prime} \neq i$. Meanwhile, the remaining $m(m-1) / 2-(m-1)$ classifiers are regarded as non-competent classifiers. To distinguish the competences of different binary classifiers, Galar et al. [11] proposed a distance-based relative competence weighting combination method for the OVO (DRCW-OVO) strategy in which the relative competence weight of each binary classifier is determined according to the average distance between a given test sample and the $K$ nearest neighbors of each class in the classifier's training sample set. This was a valuable attempt to consider the different competences of various binary classifiers in the OVO strategy. However, in some situations, it is potentially insufficient to determine the relative competence weight of a binary classifier based on the $K$ nearest neighbors of each class in the training sample set. For example, the relative competence weight of a binary classifier would be greatly affected by the outliers in the training set if they are selected as nearest neighbors, which would reduce the accuracy of the classification results. In this paper, an improved OVO strategy is proposed wherein the relative competence weight of a binary classifier is based not only on the $K$ nearest neighbors but also on the class center of each class in the classifier's training set. The improved OVO strategy is described next in Section 2.2. 


\subsection{Development of the improved OVO strategy}

In this section, an improved OVO strategy is proposed. First, the related notations and variables require introduction. Let $C=\left\{C_{1}, C_{2}\right.$, ?,$\left.C_{m}\right\}$ denote a set of $m$ classes of training samples, where $C_{i}$ denotes the $i$ th class of training samples, ${ }^{i=1,2, \text { ? }, m}$. Let $T_{i}=\left\{T_{i 1}, T_{i 2}, \mathbf{?}, T_{i q_{i}}\right\}$ denote the set of training samples of class $C_{i}$, where $T_{i h}=\left(t_{i h}^{1}, t_{i h}^{2}, \mathbf{?}, t_{i h}^{n}\right)$ denotes the $h_{\text {th training sample of class }} C_{i}$, and $t_{i h}^{j}$ denotes the value of $T_{i h}$ corresponding to the $j$ th feature, $i=1,2, ?, m, j=1,2, ?, n, h=1,2, ?, q_{i}$. In accordance with the training samples, $m(m-1) / 2$ training sample sets can be constructed, where each training sample set is the union of training samples regarding two classes, i.e., $T_{i} \mathbb{?}_{i^{\prime}}=T_{i^{\prime}} \sqrt{?}, i, i^{\prime}=1,2, ?, m$, $i \neq i^{\prime}$. Then, in accordance with the $m(m-1) / 2$ training sample sets, $m(m-1) / 2$ binary classifiers can be obtained using a machine learning algorithm. Let $V=\left(v^{1}, v^{2}, \mathbf{?}, v^{n}\right)$ denote a test sample, where $v^{j}$ denotes the value of $V$ regarding the ${ }^{j}$ th feature, $j=1,2, ?, n$. To identify the class of test sample $V, m(m-1) / 2$ binary classifiers are respectively applied, and a score matrix $R=\left(r_{i i^{\prime}}\right)_{m \times m}$ is determined according to the results of the $m(m-1) / 2$ binary classifiers. With respect to $T_{i h}$ and $R, i=1,2$, ?, $m, h=1,2$, ?,$q_{i}$, an improved OVO strategy is proposed to determine the final classification result of test sample $V$. The improved OVO strategy can be divided into five steps, described as follows:

Step 1. Determining the center of the training samples of each class.

Let $\bar{T}_{i}=\left(\overline{t_{i}^{1}}, \overline{t_{i}^{2}}\right.$, ?, $\left.\bar{t}_{i}^{n}\right)$ denote the center of the training samples of class $C_{i}$, where $\overline{t_{i}^{j}}$ denotes the value of $\bar{T}_{i}$ regarding the ${ }^{j}$ th feature, ${ }^{i=1,2, ?, m}, j=1,2, ?, n$. The value of $\overline{t_{i}^{j}}$ can be calculated by averaging the corresponding feature values of the training samples of class $C_{i}$, i.e.,

$$
\overline{t_{i}^{j}}=\sum_{h=1}^{q_{i}} t_{i h}^{j} / q_{i}, i=1,2, \mathbf{?}, m, j=1,2, \mathbf{?}, n \text {. }
$$

Thus, the center of training samples of class $C_{i}$ can be represented by 


$$
\bar{T}_{i}=\left(\sum_{h=1}^{q_{i}} t_{i h}^{1} / q_{i}, \sum_{h=1}^{q_{i}} t_{i h}^{2} / q_{i}, \mathbf{?}, \sum_{h=1}^{q_{i}} t_{i h}^{n} / q_{i}\right), i=1,2 \text {, ?, } m \text {. }
$$

Step 2. Calculating the distance between the test sample and the center of each class.

Let $d_{i}^{C}$ denote the distance between $V=\left(v^{1}, v^{2}, \mathbf{?}, v^{n}\right)$ and $\overline{T_{i}}=\left(\overline{t_{i}^{1}}, \overline{t_{i}^{2}}, \mathbf{?}, \overline{t_{i}^{n}}\right) . d_{i}^{C}$ can be calculated by Eq. (2), i.e.,

$$
d_{i}^{C}=\sqrt{\sum_{j=1}^{n}\left(v^{j}-\overline{t_{i}^{j}}\right)^{2}} \quad, \quad i=1,2, \boldsymbol{?}, m \text {. }
$$

In the present study, Euclidean distance is used because the texts are converted into term feature vectors with numerical feature values using the bag-of-words (BOW) model. When the OVO strategy is applied to other classification problems, the feature values can be interval numbers [45], fuzzy numbers [23, 24], Type-2 fuzzy numbers [26, 31] or fuzzy random numbers [27], and different formulae can be used to calculate distance. For example, the Heterogeneous Value Difference Metric (HVDM) can be used to calculate distance for nominal values [11].

Step 3. Calculating the average distance between the test sample and the $K$ nearest neighbors of each class.

Let $d_{i h}$ denote the distance between $V=\left(v^{1}, v^{2}, \mathbf{?}, v^{n}\right)$ and $T_{i h}=\left(t_{i h}^{1}, t_{i h}^{2}, \mathbf{?}, t_{i h}^{n}\right)$, $i=1,2, ?, m, h=1,2, ?, q_{i} . d_{i h}$ can be calculated by Eq. (3), i.e.,

$$
d_{i h}=\sqrt{\sum_{j=1}^{n}\left(v^{j}-t_{i h}^{j}\right)^{2}} \quad, i=1,2 \text {, ?, } m, h=1,2 \text {, ? }, q_{i} \text {. }
$$

According to the obtained $d_{i 1}, d_{i 2}$, ?,$d_{i q_{i}}$, a ranking of the $q_{i}$ distances can be determined as $d_{i(1)} \leq d_{i(2)} \leq ? \leq d_{i\left(q_{i}\right)}$, where $d_{i(h)}$ denotes the $h$ th smallest of the $q_{i}$ distances, $i=1,2, ?, m, h=1,2, \mathbf{?}, q_{i}$. Therefore, the average distance between $V$ and the $K$ nearest neighbors of class $C_{i}$ can be calculated by Eq. (4), i.e.,

$$
\bar{d}_{i}^{K}=\sum_{t=1}^{K} d_{i(t)} / K, i=1,2, \mathbf{?}, m \text {, }
$$

where $K \in\left\{1,2, \mathbb{?}, q_{i}\right\}$ is a previously determined parameter.

Step 4. Calculating the relative competence weight of each classifier. 
Let $w_{i i^{\prime}}$ denote the relative competence weight of the classifier trained by the training sample set of classes $C_{i}$ and $C_{i^{\prime}}, i, i^{\prime}=1,2$, ?, $m, i \neq i^{\prime}$. According to the obtained $d^{C}$ and $\bar{d}_{i}^{K}, w_{i i^{\prime}}$ is defined by Eq. (5), i.e.,

$$
w_{i i^{\prime}}=\frac{\left(d_{i^{\prime}}^{C}\right)^{2}}{\left(d_{i^{\prime}}^{C}\right)^{2}+\left(d_{i}^{C}\right)^{2}} \times \frac{\left(\bar{d}_{i^{\prime}}^{K}\right)^{2}}{\left(\bar{d}_{i^{\prime}}^{K}\right)^{2}+\left(\bar{d}_{i}^{K}\right)^{2}}, i, i^{\prime}=1,2, \mathbf{?}, m, i \neq i^{\prime} .
$$

Step 5. Determining the final classification result of the test sample.

A weighted score matrix $R^{w}=\left(r_{i i^{\prime}}^{w}\right)_{m \times m}$ can be obtained in accordance with the score matrix $R=\left(r_{i i^{\prime}}\right)_{m \times m}$ and the relative competence weights $w_{i i^{\prime}}, i, i^{\prime}=1,2, ?, m, i \neq i^{\prime}$, where

$$
r_{i i^{\prime}}^{w}=r_{i i^{\prime}} \times w_{i i^{\prime}}, i, i^{\prime}=1,2, ?, m, i \neq i^{\prime} \text {. }
$$

The final classification result of test sample $V$ can be determined by Eq. (7), i.e.,

$$
\operatorname{Class}(V)=\arg \max _{i=1,2, \text { ?] } m} \sum_{\substack{i=1 \\ i^{\prime} \neq i}}^{m} r_{i i^{\prime}}^{w},
$$

where $\operatorname{Class}(V)$ denotes the classification result of test sample $V, \operatorname{Class}(V) \in$ $\left\{C_{1}, C_{2}\right.$, ?,$\left.C_{m}\right\}$.

\subsection{An illustrative example}

In this section, an example is given to clearly illustrate the improved OVO strategy.

$$
\text { Let } C=\left\{C_{1}, C_{2}, C_{3}, C_{4}\right\} \text { denote a set of four classes of training samples, where } C_{i} \text { denotes }
$$
the $i$ th class of training samples, $i=1,2,3,4$. Six $(4 \times(4-1) / 2=6)$ training sample sets are constructed corresponding to the training samples of the four classes $C_{1}, C_{2}, C_{3}, C_{4}$, where each training sample set is the union of training samples of two classes. Then, a binary classifier is determined for each training sample set, i.e., six binary classifiers are obtained corresponding to the six training sample sets used in the present study. Let $V=\left(v^{1}, v^{2}, ? ?, v^{n}\right)$ denote a test sample, where $v^{j}$ denotes the value of $V$ pertaining to the $j$ th feature, $j=1,2, \mathbf{?}, n$. The six binary classifiers are used to respectively identify the class of test sample $V$. Then, in accordance with the results of the six binary classifiers of test sample $V$, the score matrix $R$ is obtained. Here, we suppose the score matrix is 


$$
R=\left[\begin{array}{cccc}
- & 0.55 & 0.49 & 0.95 \\
0.45 & - & 0.76 & 0.90 \\
0.51 & 0.24 & - & 0.98 \\
0.05 & 0.10 & 0.02 & -
\end{array}\right]
$$

As per Eq. (1), the centers of the four sample classes are calculated and denoted as $\overline{T_{1}}, \overline{T_{2}}$, $\overline{T_{3}}$ and $\bar{T}_{4}$. As per Eq. (2), the distance between test sample $V$ and the centers of $\bar{T}_{1}, \overline{T_{2}}, \overline{T_{3}}$ and $\overline{T_{4}}$ are respectively calculated. Here, we suppose that $d_{1}^{C}=1, d_{2}^{C}=2, d_{3}^{C}=2.2$ and $d_{4}^{C}=2.5$. he average distance between test sample $V$ and the $K$ nearest neighbors of each class is calculated next using Eqs. (3) and (4). Here, we suppose that $\bar{d}_{1}^{K}=0.5, \bar{d}_{2}^{K}=0.4$, $\bar{d}_{3}^{K}=0.9$ and $\bar{d}_{4}^{K}=1.4$. The relative competence weight of each classifier can be obtained using Eq. (5), i.e., $w_{12}=0.3122, w_{13}=0.6333, w_{14}=0.7645, w_{23}=0.4572, w_{24}=0.5637$, $w_{34}=0.3988, w_{21}=0.1220, w_{31}=0.0404, w_{41}=0.0156, w_{32}=0.0746, w_{24}=0.0295$ and $w_{43}=0.1276$. According to the score matrix $R$ and the relative competence weights $w_{i i^{\prime}}$, $i, i^{\prime}=1,2,3,4, i \neq i^{\prime}$, a weighted score matrix $R^{w}=\left(r_{i i^{\prime}}^{w}\right)_{m \times m}$ can be obtained using Eq.(6), i.e.,

$$
R^{w}=\left[\begin{array}{cccc}
- & 0.1717 & 0.3103 & 0.7263 \\
0.0549 & - & 0.3475 & 0.5074 \\
0.0206 & 0.0179 & - & 0.3908 \\
0.0008 & 0.0029 & 0.0026 & -
\end{array}\right]
$$

Finally, using Eq. (7), the classification result of test sample $V$ can be determined, i.e., $\operatorname{Class}(V)=C_{1}$.

\section{The proposed method for multi-class sentiment classification}

A method for multi-class sentiment classification is proposed in this section by applying the improved OVO strategy shown in Section 2. First, the framework of the proposed method is given in Section 3.1, which includes two stages: 1) selecting important text features using the information gain (IG) algorithm, and 2) identifying the sentiment classes of texts based on the improved OVO strategy and the SVM algorithm. Using this framework, a description of the two stages of the proposed method are given in Section 3.2 and Section 3.3. 


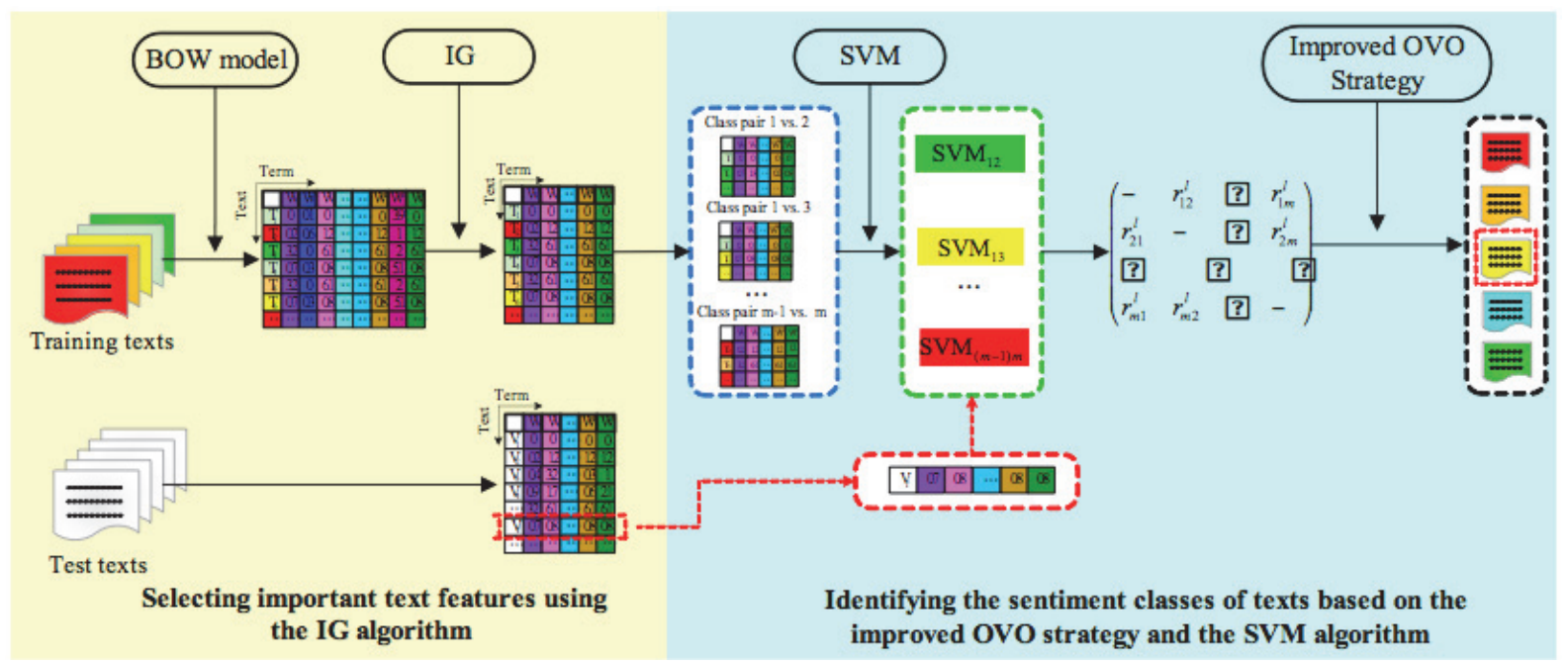

Fig. 1. Framework of the proposed method.

The framework of the proposed method is shown in Fig. 1, which shows the method's two stages: 1) selecting important text features using the IG algorithm and 2) identifying the sentiment classes of texts based on the improved OVO strategy and the SVM algorithm. In the first stage, according to the BOW model, a training text is represented by a feature vector, where each feature represents a term in the texts. To reduce the number of features, the IG algorithm is used to select important features. In the second stage, $m(m-1) / 2$ training text sets are constructed, where each training text set is the union of the training texts of two sentiment classes. Next, $m(m-1) / 2$ SVM classifiers are trained based on the $m(m-1) / 2$ training text sets regarding different pairs of sentiment classes. To identify the sentiment class of a test text, a score matrix is constructed using the results of the $m(m-1) / 2 \mathrm{SVM}$ classifiers, and the final sentiment class of the test text is determined based on a score matrix using the improved OVO strategy. Detailed descriptions of the first and second stages of the framework in Fig. 1 are given in Section 3.2 and Section 3.3, respectively.

\subsection{Selecting important text features using the IG algorithm}

Because the text terms cannot be directly identified by feature selection and machine learning algorithms, the texts are first converted into term feature vectors, and the important features (terms) of the texts are then selected using the IG algorithm. The process of selecting the important text features using the IG algorithm can be further divided into two segments: 1) converting the texts into term feature vectors, and 2) selecting the important features using 
the IG algorithm. A description of each segment follows.

\subsubsection{Converting the texts into term feature vectors}

Let $S C=\left\{S C_{1}, S C_{2}\right.$, ?,$\left.S C_{m}\right\}$ denote $m$ sentiment classes of texts, where $S C_{i}$ denotes the $i$ th sentiment class, $i=1,2$, 田, $m$. Let $T=\left\{T_{1}, T_{2}\right.$, 囵, $\left.T_{m}\right\}$ denote a set of training texts, where

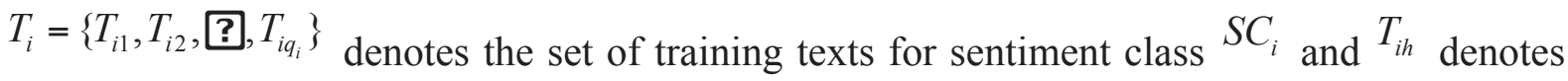
the $h$ th training text for sentiment class $S C_{i}, i=1,2$, ? $, m, h=1,2$, ?,$q_{i}$. Using the BOW model, the training texts are converted into term feature vectors as shown in Table 1.

\section{Table 1}

Term feature vectors.

\begin{tabular}{|c|c|c|c|c|c|}
\hline & Text & $t^{1}$ & $t^{2}$ & $\ldots$ & $t^{n^{t}}$ \\
\hline \multirow[t]{4}{*}{$T_{1}$} & $T_{11}$ & $\mu_{11}^{1}$ & $\mu_{11}^{2}$ & $\ldots$ & $\mu_{11}^{n^{t}}$ \\
\hline & $T_{12}$ & $\mu_{12}^{1}$ & $\mu_{12}^{2}$ & $\ldots$ & $\mu_{12}^{n^{t}}$ \\
\hline & [' & [' & {[} & {[} & {[} \\
\hline & $T_{1 q_{1}}$ & $\mu_{1 q_{1}}^{1}$ & $\mu_{1 q_{1}}^{2}$ & $\ldots$ & $\mu_{1 q_{1}}^{n^{t}}$ \\
\hline [ & [ & {[} & {[} & {[} & E \\
\hline \multirow[t]{4}{*}{$T_{m}$} & $T_{m 1}$ & $\mu_{m 1}^{1}$ & $\mu_{m 1}^{2}$ & $\ldots$ & $\mu_{m 1}^{n^{t}}$ \\
\hline & $T_{m 2}$ & $\mu_{m 2}^{1}$ & $\mu_{m 2}^{2}$ & $\ldots$ & $\mu_{m 2}^{n^{t}}$ \\
\hline & {[} & {[} & E & {[} & {[} \\
\hline & $T_{m q_{m}}$ & $\mu_{m q_{m}}^{1}$ & $\mu_{m q_{m}}^{2}$ & $\ldots$ & $\mu_{m q_{m}}^{n^{t}}$ \\
\hline
\end{tabular}

In Table $1, n^{t}$ denotes the number of terms (features) and $\mu_{i h}^{j}$ denotes the weight of term $t^{j}$ for representing the sentiment class of text $T_{i h}, i=1,2$, ? $, m, j=1,2$, ?, $n^{t}, h=1,2$, ?,$q_{i}$. Previous studies have shown that term frequency-inverse document frequency (TF-IDF) [1] is an efficient approach for determining weight $\mu_{i h}^{j}, i=1,2$, ?, $m, j=1,2$, ? $, n^{t}, h=1,2$, ?,$q_{i}$. According to TF-IDF [1], the formula for calculating $\mu_{i h}^{j}$ can be represented by Eq. (8), i.e.,

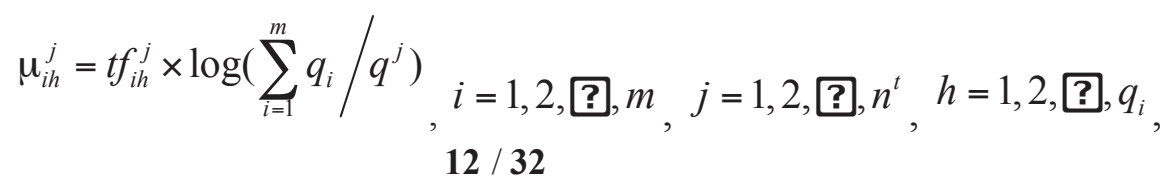


where $t f_{i h}^{j}$ is the frequency of term $t^{j}$ in text $T_{i h}, \sum_{i=1}^{m} q_{i}$ is the total number of texts of different sentiment classes and $q^{j}$ denotes the number of texts that include term $t^{j}$, $i=1,2, ?, m, j=1,2, ?, n^{t}, h=1,2, ?$, $q_{i}$. According to Eq. (8), each text $T_{i h}$ can be represented by a feature vector of terms $t^{1}, t^{2}, \mathbf{?}, t^{n^{t}}$, i.e., $T_{i h}=\left(\mu_{i h}^{1}, \mu_{i h}^{2}\right.$, ?,$\left.\mu_{i h}^{n^{t}}\right), i=1,2, ?, m$, $j=1,2, ?, n^{t}$.

\subsubsection{Selecting the important features using the IG algorithm}

Although the texts can be converted into term feature vectors using the BOW model, the dimensionality of vector $T_{i h}=\left(\mu_{i h}^{1}, \mu_{i h}^{2}, ?, \mu_{i h}^{n^{t}}\right)$ is potentially enormous, i.e., the value of $n^{t}$ is large if the number of different terms in the texts is large. An enormous number of features (terms) implies the presence of noisy and irrelevant features. Thus, to improve the efficiency and accuracy of sentiment classification, a feature selection algorithm, such as the mutual information, IG, CHI statistic or document frequency algorithm, is typically used to reduce the dimensionality of term feature vectors [37, 47]. Given a set of features (terms), feature selection algorithms can be used to select the important features (terms). According to Tan and Zhang [37] and Yang and Pedersen [47], the IG algorithm is one of the most effective feature selection algorithms for sentiment classification. Thus, in the present paper, the IG algorithm is used to select the important features (terms).

The IG algorithm is one of the most widely used feature selection algorithms for sentiment classification [37, 47]. In the IG algorithm, the importance of term $t^{j}$ is measured based on the reduction of uncertainty if the value of $t^{j}$ is known, i.e., the IG value of term $t^{j}$ is measured by the difference between the uncertainty of text $T_{i h}$ with and without $t^{j}$ [42]. Entropy is used to measure the uncertainty of text $T_{i h}$, and the IG value of $t^{j}$ is then calculated using Eq. (9), i.e.,

$$
\begin{aligned}
\operatorname{IG}\left(t^{j}\right)= & -\sum_{t=1}^{m} P\left(S C_{i}\right) \log P\left(S C_{i}\right)+P\left(t^{j}\right) \sum_{t=1}^{m} P\left(S C_{i} \mid t^{j}\right) \log P\left(S C_{i} \mid t^{j}\right) \\
& +P\left(\overline{t^{j}}\right) \sum_{t=1}^{m} P\left(S C_{i} \mid \overline{t^{j}}\right) \log P\left(S C_{i} \mid \overline{t^{j}}\right), j=1,2, \text { ? }, n^{t},
\end{aligned}
$$


where $P\left(S C_{i}\right)$ denotes the probability that a text belongs to sentiment class $S C_{i}, P\left(t^{j}\right)$ denotes the probability that term $t^{j}$ occurs in the text and $P\left(\overline{t^{j}}\right)$ denotes the probability that $t^{j}$ does not occur in the text. According to the obtained $\operatorname{IG}\left(t^{j}\right), j=1,2, ?, n^{t}$, the important features (terms) can be selected by using a pre-defined number of features or a pre-defined threshold of IG values. Here, we suppose that the number of selected features (terms) is $n^{f}$, $n^{f} \leq n^{t}$. Thus, text $T_{i h}$ can be represented by a feature vector with $n^{f}$ important features, i.e., $T_{i h}=\left(\varpi_{i h}^{1}, \varpi_{i h}^{2}, ?, \varpi_{i h}^{n^{f}}\right)$, where $\varpi_{i h}^{j}$ is the weight of the $j$ th selected feature for representing the sentiment class of text $T_{i h}, i=1,2, ?, m, j=1,2$, ? $, n^{f}, h=1,2$, ?, $q_{i}$.

\subsection{Identifying the sentiment classes of texts based on the improved OVO strategy and the} SVM algorithm

In accordance with the obtained $T_{i h}=\left(\varpi_{i h}^{1}, \varpi_{i h}^{2}, \boldsymbol{?}, \varpi_{i h}^{n^{f}}\right), i=1,2, ?, m, h=1,2, ? \mathbf{?}, q_{i}$, $m(m-1) / 2$ training text sets are constructed, where each training text set is the union of training texts for two sentiment classes. Next, $m(m-1) / 2$ SVM classifiers are trained using the SVM algorithm. To identify the sentiment class of a test text, a score matrix is constructed based on the results of the $m(m-1) / 2$ SVM classifiers for the test text, and the final sentiment classification result of the test text is determined based on the obtained score matrix using the improved OVO strategy. Thus, the process of identifying sentiment classes of texts using the improved OVO strategy and the SVM algorithm can be further divided into two segments: 1) training $m(m-1) / 2$ SVM classifiers, and 2) constructing a score matrix and determining the sentiment classification result of each text. A description of each segment is given below.

\subsubsection{Training $m(m-1) / 2$ SVM classifiers}

To identify the sentiment classes of texts based on the SVM algorithm, it is necessary to train multiple binary SVM classifiers with respect to different pairs of sentiment classes. First, $m(m-1) / 2$ training text sets are constructed, where each training text set is the union of training samples concerning two sentiment classes. Let $T S_{i i^{\prime}}=T_{i} \boldsymbol{?}_{i^{\prime}}=\left\{T_{i 1}, T_{i 2}, \boldsymbol{?}, T_{i q_{i}}\right\}$ 
$\left\{T_{i^{\prime} 1}, T_{i^{\prime} 2}\right.$, ?,$\left.T_{i^{\prime} q_{i}}\right\}$ denote the training text set for sentiment classes $S C_{i}$ and $S C_{i^{\prime}}$, $i, i^{\prime}=1,2, ?, m, i \neq i^{\prime}$. There are $m(m-1) / 2$ training text sets because $T S_{i i^{\prime}}=T S_{i^{\prime} i}$, $i, i^{\prime}=1,2, ?, m, i \neq i^{\prime}$. Based on the training text set $T S_{i i^{\prime}}$, the SVM algorithm is used to train a binary SVM classifier, denoted as $S V M_{i i^{\prime}}, i, i^{\prime}=1,2, ?, m, i \neq i^{\prime}$. Thus, using the $m(m-1) / 2$ training text sets, $m(m-1) / 2$ binary SVM classifiers are obtained, which are denoted $S V M_{12}, S V M_{13}, 4 S V M_{1 m}, S V M_{23}, 4, S V M_{(m-1) m}$.

3.3.2. Constructing a score matrix and determining the sentiment classification result of each text

Let $V=\left\{V_{1}, V_{2}\right.$, ?,$\left.V_{q_{v}}\right\}$ denote the set of $q_{v}$ test texts, where $V_{l}$ is the $l$ th test text, $l=1,2, ?, q_{v}$. Without any loss of generality, we consider that $V_{l}$ has been converted into a feature vector with the important features as per Section 3.2.2. Thus, test text $V_{l}$ can be

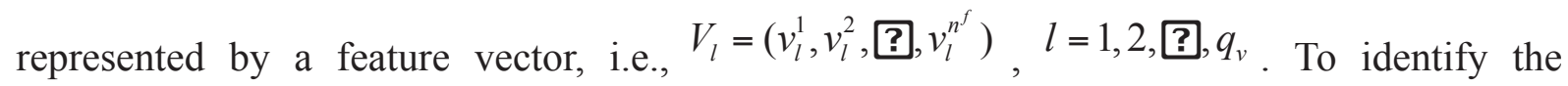
sentiment class of test text $V_{l}$, the feature vector $V_{l}=\left(v_{l}^{1}, v_{l}^{2}\right.$, ?,$\left.v_{l}^{n^{f}}\right)$ is used as input to the obtained binary classifier $S V M_{i i^{\prime}}$, and a function value for test text $V_{l}$ is obtained, denoted as $x_{l}^{i i^{\prime}}, l=1,2$, ? $, q_{v}, i, i^{\prime}=1,2$, ?, $m, i \neq i^{\prime}$. It is necessary to note that $x_{l}^{i i^{\prime}}$ is an intermediate result of classifier $S V M_{i i^{\prime}}, l=1,2, ?, q_{v}, i, i^{\prime}=1,2$, ? $, m, i \neq i^{\prime}$. In accordance with $x_{l}^{i i^{\prime}}$, the output of $S V M_{i i^{\prime}}$ for identifying the sentiment class of test text $V_{l}$ could be obtained, i.e., $\operatorname{sign}\left(x_{l}^{i i^{\prime}}\right) \in\{0,1\}$, where $\operatorname{sign}\left(x_{l}^{i i^{\prime}}\right)=1$ represents the classification result that the sentiment class of test text $V_{l}$ is $S C_{i}$, and $\operatorname{sign}\left(x_{l}^{i i^{\prime}}\right)=0$ represents the classification result that it is $S C_{i^{\prime}}$, $l=1,2, ?, q_{v}, i, i^{\prime}=1,2, ?, m, i \neq i^{\prime}$. To distinguish the confidence of different binary SVM classifiers for identifying the sentiment class of test text $V_{l}$, the confidence of the binary classifier $S V M_{i i^{\prime}}$ can be calculated according to the function values $x_{l}^{i i^{\prime}}, i, i^{\prime}=1,2$, ?, $m$, $i \neq i^{\prime}, l=1,2, ?, q_{v}$. Let $R^{l}=\left(r_{i i^{\prime}}^{l}\right)_{m \times m}$ denote the score matrix for test text $V_{l}$, where $r_{i i^{\prime}}^{l} \in[0,1]$ is the confidence of binary classifier $S V M_{i i^{\prime}}$ for identifying the sentiment class of test text $V_{l}$, $i, i^{\prime}=1,2, ?, m, i \neq i^{\prime}, l=1,2$, ?, $q_{v}$. According to Platt [30], $r_{i i^{\prime}}^{l}$ can be calculated by Eq. (10), 
i.e.,

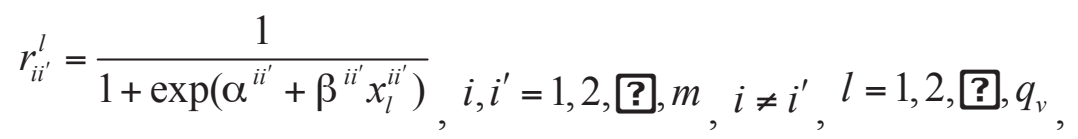

where $\alpha^{i i^{\prime}}$ and $\beta^{i i^{\prime}}$ are parameters. The values of $\alpha^{i i^{\prime}}$ and $\beta^{i i^{\prime}}$ depend on the number and categories of training texts of classifier $S V M_{i i^{\prime}}, i, i^{\prime}=1,2$, ? $, m, i \neq i^{\prime}$. The values of $\alpha^{i i^{\prime}}$ and $\beta^{i i^{\prime}}$ can be determined by maximizing the likelihood function on the training texts, i.e.,

$$
\begin{gathered}
L\left(\alpha^{i i^{\prime}}, \beta^{i i^{\prime}}\right)=\prod_{h=1}^{q_{i}+q_{i^{\prime}}}\left(p_{h}^{i i^{\prime}}\right)^{i i^{i i^{\prime}}}\left(1-p_{h}^{i i^{\prime}}\right)^{t_{i i^{\prime}}}{ }^{i i^{\prime}}=\frac{1, i^{\prime}=1,2, \boldsymbol{?}, m, i \neq i^{\prime},}{1+\exp \left(\alpha^{i i^{\prime}}+\beta^{i i^{\prime}} \theta_{h}^{i i^{\prime}}\right)}, i, i^{\prime}=1,2, \mathbf{?}, m, i \neq i^{\prime}, \\
t_{i}^{i i^{\prime}}=\frac{q_{i}+1}{q_{i}+2}, i, i^{\prime}=1,2, \mathbf{?}, m, i \neq i^{\prime}, \\
t_{i^{\prime}}^{i i^{\prime}}=\frac{1}{q_{i^{\prime}}+2}, i, i^{\prime}=1,2, \mathbf{?}, m, i \neq i^{\prime} .
\end{gathered}
$$

In Eqs. (11)-(14), ${ }^{\theta_{h}^{i i^{\prime}}}$ is the score value of the $h$ th training text in set $T S_{i i^{\prime}}=T_{i} \mathbb{P}_{i^{\prime}} \cdot \theta_{h}^{i i^{\prime}}$ can be calculated by a pre-trained classifier [30]. Meanwhile, the value of ${ }^{r^{\prime} i}$ can be calculated by Eq. (15), i.e.,

$$
r_{i^{\prime} i}^{l}=1-r_{i i^{\prime}}^{l}=1-\frac{1}{1+\exp \left(\alpha^{i i^{\prime}}+\beta^{i i^{\prime}} x_{l}^{i i^{\prime}}\right)}, i, i^{\prime}=1,2, \boldsymbol{?}, m, i \neq i^{\prime}, l=1,2 \text {, ? }, q_{v} .
$$

According to Eqs. (10)-(15), the confidences of the $m(m-1) / 2$ binary SVM classifiers for identifying sentiment class of test text $V_{l}$ can be obtained, i.e., the score matrix $R^{l}=\left(r_{i i^{\prime}}^{l}\right)_{m \times m}$, $l=1,2, ?, q_{v}$, can be constructed. According to the obtained score matrix $R^{l}=\left(r_{i i^{\prime}}^{l}\right)_{m \times m}$, the sentiment classification result of test text $V_{l}$ can be determined using the improved OVO strategy proposed in Section 2.2, i.e., Eqs. (1)-(7), $l=1,2, ?$ ?, $q_{v}$.

\section{Experimental framework}

We present several experimental studies in Section 5 to illustrate the effectiveness of the proposed method. In this section, the experimental framework is introduced, which includes 
three segments: 1) experimental datasets, 2) parameter configuration and 3) performance measures and statistical tests. A detailed description of each segment is given below.

\subsection{Experimental datasets}

We conducted the experiments on the sentiment scale datasets in the Movie Review Data set (http://www.cs.cornell.edu/people/pabo/movie-review-data/), which include four corpora of movie reviews. In each of the four corpora, both a sentiment class based on 3-class sentiment classification and a sentiment class based on 4-class sentiment classification are simultaneously assigned to each movie review [29]. Thus, the four corpora can be further divided into eight data subsets, denoted Data subset 1 , Data subset $2, \ldots \ldots$, Data subset 8 . The related information of the eight data subsets is shown in Table 2. In Table 2, \#Cl. denotes the number of sentiment classes that have been assigned to the texts, \#T denotes the number of texts, $\# \mathrm{~F}$ denotes the number of text features and $\mathrm{C}_{1}, \mathrm{C}_{2}, \mathrm{C}_{3}$ and $\mathrm{C}_{4}$ denote the number of texts belonging to the respective sentiment classes. For example, in Data subset 1, there are 1770 texts with 26439 text features, and three types of sentiment classes have been assigned to the 1770 texts, i.e., $C_{1}, C_{2}$ and $C_{3}$. The number of texts belonging to sentiment classes $C_{1}, C_{2}$ and $\mathrm{C}_{3}$ are 413,648 and 709 , respectively.

Table 2

Related information about the experimental datasets.

\begin{tabular}{lllllllll}
\hline Data set & Data subset & $\#$ Cl. & $\#$ T & $\# \mathrm{~F}$ & $\mathrm{C}_{1}$ & $\mathrm{C}_{2}$ & $\mathrm{C}_{3}$ & $\mathrm{C}_{4}$ \\
\hline & Data subset 1 & 3 & 1770 & 26439 & 413 & 648 & 709 & \\
& Data subset 2 & 3 & 902 & 25897 & 239 & 349 & 314 & \\
& Data subset 3 & 3 & 1307 & 31188 & 186 & 491 & 630 & \\
Movie & Data subset 4 & 3 & 1027 & 19237 & 359 & 427 & 241 & \\
Review & Data subset 5 & 4 & 1770 & 26439 & 191 & 526 & 766 & 287 \\
Data & Data subset 6 & 4 & 902 & 25897 & 115 & 295 & 334 & 158 \\
& Data subset 7 & 4 & 1307 & 31188 & 138 & 292 & 596 & 281 \\
& Data subset 8 & 4 & 1027 & 19237 & 171 & 440 & 302 & 114 \\
\hline
\end{tabular}

10-fold cross validation was used to evaluate the performance of the proposed method. First, each data subset in Table 2 was divided into 10 groups of similar size and distribution. Then, the union of 9 groups was used as a training set, and the remaining group was used as a test set. This process was repeated ten times, such that each group was used once as a test set. 
The average test accuracy of the ten times was regarded to be the accuracy of the 10 -fold cross validation.

\subsection{Parameter configuration}

The data mining toolkit WEKA (Waikato Environment for Knowledge Analysis) version 3.7.0 (http://www.cs. waikato.ac.nz/ml/weka/) was used in the experiments. WEKA is a collection of various feature selection and machine learning algorithms for data mining tasks $[16]$.

The basic algorithms used in the present study, the IG and SVM algorithms, were implemented by the Info Gain Attribute Eval and LIBSVM modules, respectively (Library for Support Vector Machines) (http://www.csie.ntu.edu.tw/ cjlin/libsvm/index.html\# nuandone). The parameter settings of the Info Gain Attribute Eval and LIBSVM modules are given below.

1) Parameter settings of the Info Gain Attribute Eval module: Generate ranking=True, Num to select $=-1$, Threshold $=0$;

2) Parameter settings of the LIBSVM module: Cost parameter=1.0, Tolerance parameter $=0.001$, Kernel type $=$ radial basis function, Degree $=3$, Probability estimates $=$ True.

It can be seen from the parameter settings of the Info Gain Attribute Eval module that Threshold $=0$. This indicates that a feature (term) will be selected if its IG value is greater than 0 . Thus, the number of selected features depends on the IG values of the features (terms).

\subsection{Performance measures and statistical tests}

To evaluate the performance of proposed method, average accuracy (AA), weighted average recall (WAR), weighted average precision (WAP) and weighted average F-Measure (WAF-M) were calculated [7, 16]. To calculate AA, WAR, WAP and WAF-M, a confusion

matrix was constructed as shown in Table 3 . In Table $3,{ }^{c_{i i^{\prime}}}$ denotes the number of texts that whose actual sentiment class are $S C_{i}$ and are classified into sentiment class $S C_{i^{\prime}}$, $i, i^{\prime}=1,2, ?, m$.

\section{Table 3}

Confusion matrix for multi-class sentiment classification. 


\begin{tabular}{|c|c|c|c|c|c|}
\hline & & \\
\hline & & $S C_{1}$ & $S C_{2}$ & ᄂ? & $S C_{m}$ \\
\hline \multirow{4}{*}{ Actual sentiment class } & $S C_{1}$ & $c_{11}$ & $c_{12}$ & Ł? & $c_{1 m}$ \\
\hline & $S C_{2}$ & $c_{21}$ & $c_{22}$ & பீ & $c_{2 m}$ \\
\hline & {[} & {[} & {[} & ? & {[} \\
\hline & $S C_{m}$ & $c_{m 1}$ & $c_{m 2}$ & U & $c_{m m}$ \\
\hline
\end{tabular}

The formulas for calculating AA, WAR, WAP and WAF-M can be respectively represented by Eqs. (16)-(19), i.e.,

$$
\begin{gathered}
\mathrm{AA}=\frac{\sum_{i=1}^{m} c_{i i}}{\sum_{i=1}^{m} \sum_{i=1}^{m} c_{i i^{\prime}}} \\
\mathrm{WAR}=\sum_{i=1}^{m} c w_{i} \times c_{i i} / \sum_{i=1}^{m} c_{i i^{\prime}}, \\
\mathrm{WAP}=\sum_{i=1}^{m} c w_{i} \times c_{i i} / \sum_{i=1}^{m} c_{i^{\prime} i}, \\
\mathrm{WAF}-\mathrm{M}=2 \times \sum_{i=1}^{m} c w_{i} \times \frac{c_{i i} / \sum_{i=1}^{m} c_{i i^{\prime}} \times c_{i i} / \sum_{i=1}^{m} c_{i^{\prime} i}}{c_{i i} / \sum_{i=1}^{m} c_{i i^{\prime}}+c_{i i} / \sum_{i=1}^{m} c_{i^{\prime} i}},
\end{gathered}
$$

where ${ }^{c w_{i}}$ denotes the weight of the test texts belonging to sentiment class $S C_{i}, i=1,2, ?, m$. The ${ }^{c w_{i}}$ can be calculated by Eq. (20), i.e.,

$$
c w_{i}=\frac{\sum_{i=1}^{m} c_{i i^{\prime}}}{\sum_{i=1}^{m} \sum_{i=1}^{m} c_{i i^{\prime}}}=\frac{q_{i}}{\sum_{i=1}^{m} q_{i}}, i=1,2, \boldsymbol{?}, m \text {. }
$$

To verify the significance of our experimental results, non-parametric tests were used. As per the literature [8, 13], the Wilcoxon test [43] was applied. Let $\alpha_{M}^{b}$ and $\alpha_{M^{\prime}}^{b}$ respectively denote the performance of methods $M$ and $M^{\prime}$ for identifying the sentiment classes of the texts in the $b$ th data subset, $b=1,2,0,8$. In this study, $\alpha_{M}^{b}$ and $\alpha_{M^{\prime}}^{b}$ refer to AA, WAR, WAP and WAF-M, and $M$ and $M^{\prime}$ refer to the different methods used for multi-class sentiment classification, including the existing and proposed methods with different values of $K$. Let $d_{M M^{\prime}}^{b}$ denote the difference between $\alpha_{M}^{b}$ and $\alpha_{M^{\prime}}^{b}$, i.e., 


$$
d_{M M^{\prime}}^{b}=\alpha_{M}^{b}-\alpha_{M^{\prime}}^{b}, b=1,2, ?, 8 .
$$

The experiments were conducted with eight data subsets, thus there were eight $d_{M M^{\prime}}^{b}$ for methods $M$ and $M^{\prime}$, i.e., $d_{M M^{\prime}}^{1}, d_{M M^{\prime}}^{2}$, ?, $d_{M M^{\prime}}^{8}$. Let $\left|d_{M M^{\prime}}^{(1)}\right| \leq\left|d_{M M^{\prime}}^{(2)}\right| \leq ? \leq\left|d_{M M^{\prime}}^{(8)}\right|$ denote a ranking of the absolute values of $d_{M M^{\prime}}^{1}, d_{M M^{\prime}}^{2}$, ?,$d_{M M^{\prime}}^{8}$ from the smallest to the greatest value. Let $p_{M M^{\prime}}^{b}$ denote the ranking position of $d_{M M^{\prime}}^{b}$ in the ranking $\left|d_{M M^{\prime}}^{(1)}\right| \leq\left|d_{M M^{\prime}}^{(2)}\right| \leq \mathbf{?} \leq\left|d_{M M^{\prime}}^{(8)}\right|$, i.e., $p_{M M^{\prime}}^{b} \in\{1,2, \boldsymbol{?}, 8\}$ and $p_{M M^{\prime}}^{b} \neq p_{M M^{\prime}}^{b^{\prime}}$ if $b \neq b^{\prime}$. Let $I_{M M^{\prime}}^{b}$ denote an indication variable of $d_{M M^{\prime}}^{b}$, $b=1,2, ?, 8$. As per the Wilcoxon test [43], the value of $I_{M M^{\prime}}^{b}$ can be found using Eq. (22), i.e.,

$$
I_{M M^{\prime}}^{b}= \begin{cases}1, & \text { if } d_{M M^{\prime}}^{b}>0, \\ 0.5, & \text { if } d_{M M^{\prime}}^{b}=0, \\ 0, & \text { if } d_{M M^{\prime}}^{b}<0, \quad b=1,2, ?, 8,\end{cases}
$$

where $1,0.5$ and 0 are the coefficients of $p_{M M^{\prime}}^{b}$ with respect to $\alpha_{M}^{b}>\alpha_{M^{\prime}}^{b}, \alpha_{M}^{b}=\alpha_{M^{\prime}}^{b}$ and $\alpha_{M}^{b}<\alpha_{M^{\prime}}^{b}$, respectively.

Let $R_{M M^{\prime}}^{+}$be a score indicating the degree to which method $M$ outperforms method $M^{\prime}$, and $R_{M M^{\prime}}^{-}$denote a score indicating the degree to which method $M^{\prime}$ outperforms method $M$. As per the Wilcoxon test [43], $R_{M M^{\prime}}^{+}$and $R_{M M^{\prime}}^{-}$can be calculated using Eqs. (23) and (24), respectively, i.e.,

$$
\begin{gathered}
R_{M M^{\prime}}^{+}=\sum_{b=1}^{8} p_{M M^{\prime}}^{b} I_{M M^{\prime}}^{b}, \\
R_{M M^{\prime}}^{-}=\sum_{b=1}^{8} p_{M M^{\prime}}^{b}\left(1-I_{M M^{\prime}}^{b}\right) .
\end{gathered}
$$

Let $T_{M M^{\prime}}=\min \left\{R_{M M^{\prime}}^{+}, R_{M M^{\prime}}^{-}\right\}$denote the value for testing the significance of the difference between methods $M$ and $M^{\prime}$. In accordance with the obtained $T_{M M^{\prime}}$ and the number of data subsets, the significance of the difference between $M$ and $M^{\prime}$ can be verified by consulting the Wilcoxon rank sum table.

\section{Experimental studies}


In this section, we describe the experimental studies conducted to illustrate the effectiveness of the proposed method. First, to analyze the effect of the value of $K$ on the sentiment classification results, experiments with different values of $K$ were conducted and analyzed. Next, comparisons between the proposed method and the existing methods for multi-class sentiment classification are presented. Finally, we examine the performance of the improved and existing OVO strategies on multi-class sentiment classification.

\subsection{Analyzing the effects of different values of $K$}

In the proposed method, the $K$ nearest neighbors and the class center of each class in the training text set are used to determine the relative competence weight of each binary SVM classifier. The value of $K$ would affect the performance of the proposed method; thus, to analyze the effect of the value of $K$, several values of $K$ were selected, i.e., $K=1,5,10$ and 20 . In addition, the situation where $K=-1$, i.e., all the training texts of each sentiment class were used to determine the relative competence weight of the classifier, was also considered.

Using 10-fold cross validation, the proposed method was applied to the eight data subsets. The values of AA, WAR, WAP and WAF-M using different values of $K$ are shown in Table 4. The average AA, WAR, WAP and WAF-M values that resulted from using different values of $K$ are shown in Fig. 2. It can be seen from Fig. 2 that the results using $K=1,5,10$ and 20 are stable, and the results using $K=-1$ are slightly inferior to these. As stated in to Section 4.3, Wilcoxon tests were conducted to obtain more meaningful conclusions. Table 5 shows the results of the Wilcoxon tests for verifying significant pairwise differences between different values of $K$ with respect to AA, WAR, WAP or WAF-M. It can be seen from Table 5 that significant pairwise differences were not found using $K=1,5,10$ and 20 . This indicates that different values of $K$ do not substantially affect the performance of the proposed method. In addition, it can be seen from Table 5 that significant differences were found only in the cases of $K=1$ vs. $K=-1, K=10$ vs. $K=-1$ and $K=20$ vs. $K=-1$.

Table 4

Values of AA, WAR, WAP and WAF-M of the proposed method on each of the eight data subsets with different values of $K$.

\begin{tabular}{|c|c|c|c|c|c|c|c|c|c|c|}
\hline & $K$ & $\mathrm{AA}$ & WAR & WAP & WAF-M & $K$ & AA & WAR & WAP & WAF-M \\
\hline & 1 & 80.43 & 80.43 & 81.07 & 80.55 & 1 & 80.49 & 80.49 & 81.06 & 80.53 \\
\hline $\mathrm{D}$ a t a & 5 & 80.32 & 80.32 & 81.07 & 80.47 & 5 & 80.15 & 80.15 & 80.86 & 80.21 \\
\hline
\end{tabular}




\begin{tabular}{|c|c|c|c|c|c|c|c|c|c|c|c|}
\hline \multirow{3}{*}{$\begin{array}{l}\text { subset } \\
1\end{array}$} & 10 & 80.37 & 80.37 & 81.19 & 80.51 & \multirow{3}{*}{$\begin{array}{l}\text { L a I a } \\
\text { subset } 2\end{array}$} & \multirow{2}{*}{$\begin{array}{l}10 \\
20\end{array}$} & \multirow{2}{*}{$\begin{array}{l}80.04 \\
80.15\end{array}$} & \multirow{2}{*}{$\begin{array}{l}80.04 \\
80.15\end{array}$} & \multirow{2}{*}{$\begin{array}{l}80.82 \\
80.96\end{array}$} & \multirow{2}{*}{$\begin{array}{l}80.11 \\
80.23\end{array}$} \\
\hline & 20 & 80.32 & 80.32 & 81.17 & 80.46 & & & & & & \\
\hline & -1 & 80.32 & 80.32 & 81.67 & 80.52 & & -1 & 79.93 & 79.93 & 81.07 & 80.04 \\
\hline \multirow{5}{*}{$\begin{array}{l}\mathrm{D} \text { a t a } \\
\text { subset } \\
3\end{array}$} & 1 & 86.31 & 86.31 & 86.90 & 86.40 & \multirow{5}{*}{$\begin{array}{l}D \text { a } t \text { a } \\
\text { subset } 4\end{array}$} & 1 & 74.02 & 74.02 & 75.36 & 74.12 \\
\hline & 5 & 82.44 & 82.44 & 83.01 & 82.60 & & 5 & 72.83 & 72.83 & 76.25 & 73.37 \\
\hline & 10 & 86.57 & 86.57 & 87.02 & 86.60 & & 10 & 72.83 & 72.83 & 76.25 & 73.37 \\
\hline & 20 & 86.59 & 86.59 & 87.02 & 86.61 & & 20 & 72.83 & 72.83 & 76.25 & 73.37 \\
\hline & -1 & 86.49 & 86.49 & 87.05 & 86.53 & & -1 & 70.65 & 70.65 & 76.29 & 70.98 \\
\hline \multirow{5}{*}{$\begin{array}{l}\text { D a t a } \\
\text { subset } \\
5\end{array}$} & 1 & 73.59 & 73.59 & 74.61 & 73.53 & \multirow{5}{*}{$\begin{array}{l}D \text { a } t \text { a } \\
\text { subset } 6\end{array}$} & 1 & 68.14 & 68.14 & 69.12 & 67.93 \\
\hline & 5 & 73.59 & 73.59 & 74.83 & 73.47 & & 5 & 69.79 & 69.79 & 71.26 & 69.63 \\
\hline & 10 & 73.19 & 73.19 & 74.56 & 73.05 & & 10 & 69.13 & 69.13 & 70.78 & 68.94 \\
\hline & 20 & 73.03 & 73.03 & 74.53 & 72.87 & & 20 & 69.12 & 69.12 & 71.00 & 68.86 \\
\hline & -1 & 71.90 & 71.9 & 74.01 & 71.68 & & -1 & 68.13 & 68.13 & 70.46 & 67.77 \\
\hline \multirow{5}{*}{$\begin{array}{l}\text { D a t a } \\
\text { subset } \\
7\end{array}$} & 1 & 78.42 & 78.42 & 78.76 & 78.10 & \multirow{5}{*}{$\begin{array}{l}D \text { a } t \text { a } \\
\text { subset } 8\end{array}$} & 1 & 67.33 & 67.33 & 67.89 & 66.84 \\
\hline & 5 & 77.27 & 77.27 & 77.70 & 76.94 & & 5 & 71.15 & 71.15 & 73.70 & 70.11 \\
\hline & 10 & 76.81 & 76.81 & 77.32 & 76.51 & & 10 & 66.35 & 66.35 & 68.23 & 64.8 \\
\hline & 20 & 76.58 & 76.58 & 77.12 & 76.23 & & 20 & 66.29 & 66.29 & 67.64 & 65.45 \\
\hline & -1 & 76.27 & 76.27 & 77.27 & 75.84 & & -1 & 65.61 & 65.61 & 67.99 & 64.26 \\
\hline
\end{tabular}

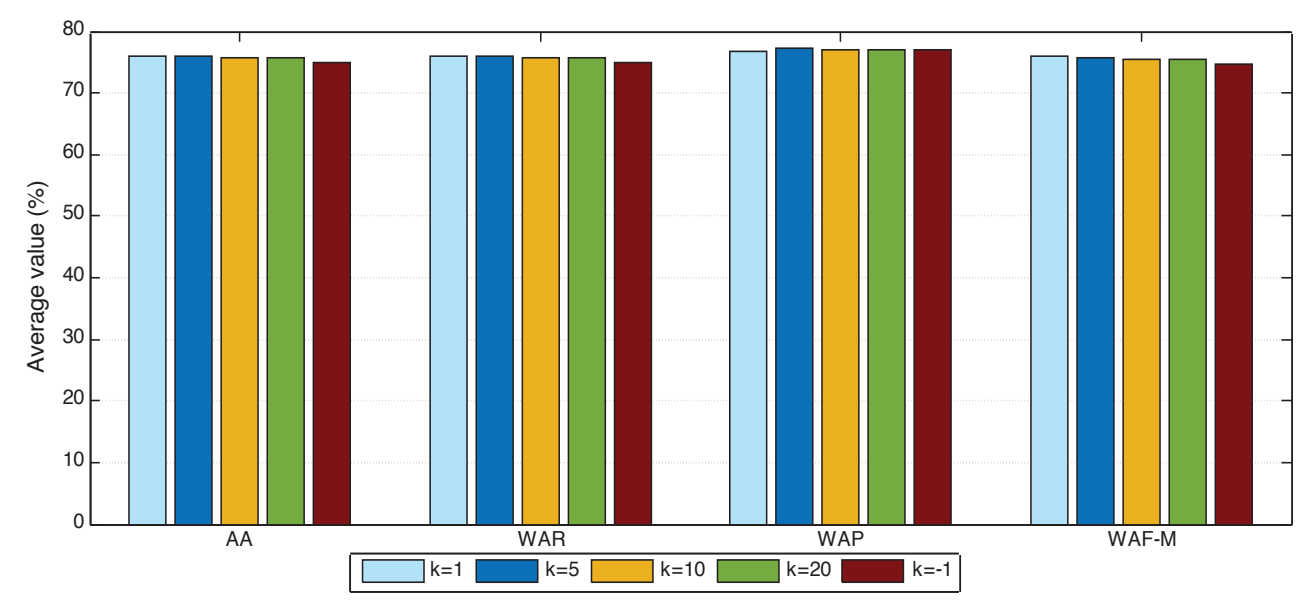

Fig. 2. Average values of AA, WAR, WAP and WAF-M with different values of $K$.

\section{Table 5}

Wilcoxon test results for verifying significant pairwise differences using different values of $K$ with respect to AA, WAR, WAP or WAF-M.

\begin{tabular}{llllll}
\hline Values of $K$ & Measure & $\mathrm{R}^{+}$ & $\mathrm{R}^{-}$ & Hypothesis & $p$-Value \\
\hline \multirow{4}{*}{1 vs. 5 } & AA & 17 & 11 & Not rejected & 0.612 \\
& WAR & 17 & 11 & Not rejected & 0.612 \\
& WAP & 11 & 17 & Not rejected & 0.612
\end{tabular}




\begin{tabular}{|c|c|c|c|c|c|}
\hline & WAF-M & 23 & 13 & Not rejected & 0.484 \\
\hline \multirow{4}{*}{1 vs. 10} & $\mathrm{AA}$ & 28 & 8 & Not rejected & 0.161 \\
\hline & WAR & 28 & 8 & Not rejected & 0.161 \\
\hline & WAP & 12 & 24 & Not rejected & 0.400 \\
\hline & WAF-M & 28 & 8 & Not rejected & 0.161 \\
\hline \multirow{4}{*}{1 vs. 20} & $\mathrm{AA}$ & 29 & 7 & Not rejected & 0.123 \\
\hline & WAR & 29 & 7 & Not rejected & 0.123 \\
\hline & WAP & 15.5 & 22.5 & Not rejected & 0.726 \\
\hline & WAF-M & 28 & 8 & Not rejected & 0.161 \\
\hline \multirow{4}{*}{1 vs. -1} & AA & 33 & 3 & Rejected for -1 at $95 \%$ & 0.036 \\
\hline & WAR & 33 & 3 & Rejected for -1 at $95 \%$ & 0.036 \\
\hline & WAP & 12.5 & 23.5 & Not rejected & 0.441 \\
\hline & WAF-M & 34 & 2 & Rejected for -1 at $95 \%$ & 0.025 \\
\hline \multirow{4}{*}{5 vs. 10} & $\mathrm{AA}$ & 21 & 7 & Not rejected & 0.237 \\
\hline & WAR & 21 & 7 & Not rejected & 0.237 \\
\hline & WAP & 20 & 8 & Not rejected & 0.310 \\
\hline & WAF-M & 21 & 7 & Not rejected & 0.237 \\
\hline \multirow{4}{*}{5 vs. 20} & $\mathrm{AA}$ & 11 & 4 & Not rejected & 0.345 \\
\hline & WAR & 11 & 4 & Not rejected & 0.345 \\
\hline & WAP & 19 & 9 & Not rejected & 0.397 \\
\hline & WAF-M & 20 & 8 & Not rejected & 0.310 \\
\hline \multirow{4}{*}{5 vs. -1} & $\mathrm{AA}$ & 22 & 6 & Not rejected & 0.176 \\
\hline & WAR & 22 & 6 & Not rejected & 0.176 \\
\hline & WAP & 22 & 14 & Not rejected & 0.575 \\
\hline & WAF-M & 16 & 12 & Not rejected & 0.735 \\
\hline \multirow{4}{*}{10 vs. 20} & AA & 21 & 7 & Not rejected & 0.237 \\
\hline & WAR & 21 & 7 & Not rejected & 0.237 \\
\hline & WAP & 13 & 8 & Not rejected & 0.600 \\
\hline & WAF-M & 16 & 12 & Not rejected & 0.735 \\
\hline \multirow{4}{*}{10 vs. -1} & AA & 36 & 0 & Rejected for -1 at $95 \%$ & 0.012 \\
\hline & WAR & 36 & 0 & Rejected for -1 at $95 \%$ & 0.012 \\
\hline & WAP & 21 & 15 & Not rejected & 0.674 \\
\hline & WAF-M & 35 & 1 & Rejected for -1 at $95 \%$ & 0.017 \\
\hline \multirow{4}{*}{20 vs. -1} & $\mathrm{AA}$ & 28 & 0 & Rejected for -1 at $95 \%$ & 0.018 \\
\hline & WAR & 28 & 0 & Rejected for -1 at $95 \%$ & 0.018 \\
\hline & WAP & 15 & 21 & Not rejected & 0.674 \\
\hline & WAF-M & 35 & 1 & Rejected for -1 at $95 \%$ & 0.017 \\
\hline
\end{tabular}

Notes: 1. Each 'Hypothesis' declares that there is no significant difference in the values of AA, WAR, WAP or WAF-M using different values of $K$. For example, the first Hypothesis is "there is no significant difference between the values of AA with $K=1$ and $K=5$ ".

2: The $p$-value indicates the significance of the differences between the values of AA, WAR, WAP or WAF-M using different values of $K$. 


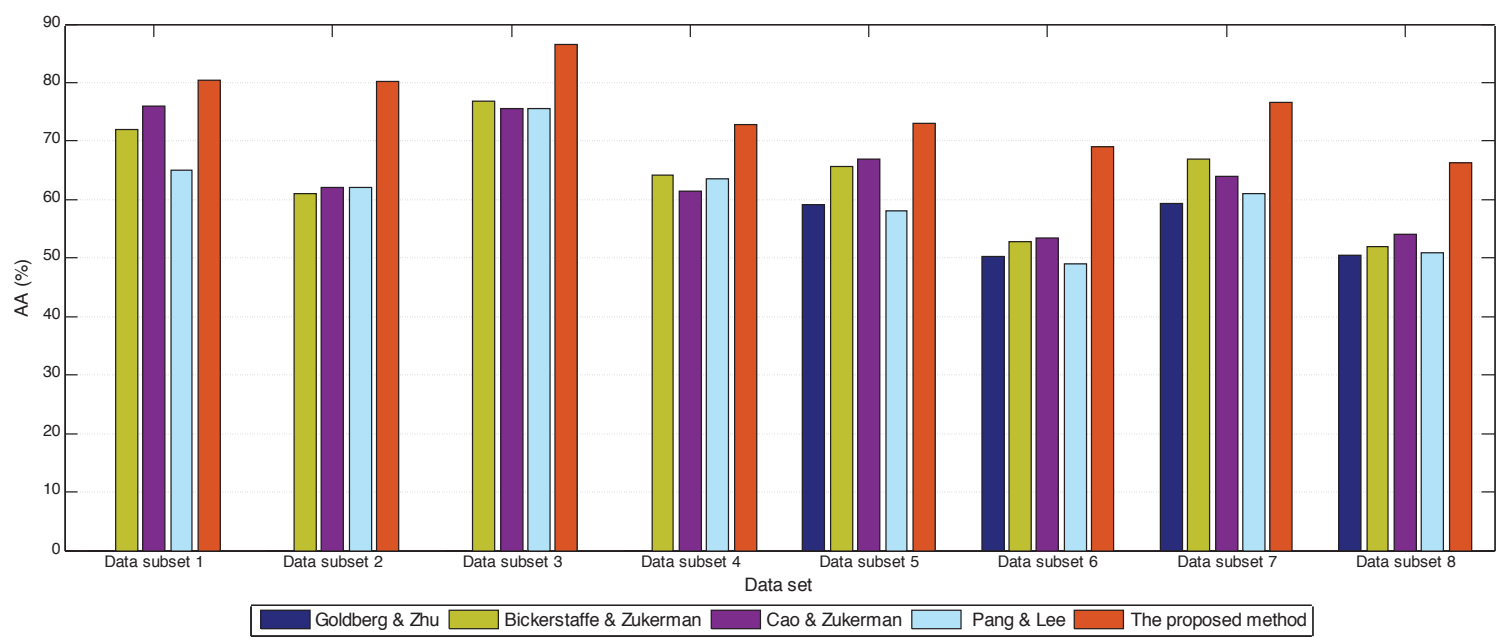

Fig. 3. AA values for sentiment classification using the previously existing and proposed methods $(K=20)$.

\subsection{Comparing the proposed method with the existing methods}

To illustrate the effectiveness of the proposed method, it was compared with several existing methods for multi-class sentiment classification. In the existing studies, several methods for multi-class sentiment classification have been proposed, in which the sentiment scale datasets in the Movie Review Data are used to illustrate the effectiveness of the existing methods. According to the sentiment classification results obtained by the existing methods and the proposed methods, the proposed method was compared with several existing methods including Bickerstaffe \& Zukerman [4], Cao \& Zukerman [5], Pang \& Lee [29], and Goldberg \& Zhu [15].

Fig. 3 shows the AA values of the sentiment classification results obtained by the existing and proposed methods. In the study of Goldberg \& Zhu [5], 3-class sentiment classification results were not considered; thus, only the 4-class sentiment classification results obtained by Goldberg \& Zhu's method are shown in Fig. 3. In addition, Fig. 3 only shows the proposed method's AA values with $K=20$ because its AA values with $K=1,5$, and 10 are higher than these values. It can be seen from Fig. 3 that the AA values of the proposed method are significantly higher than those of the existing methods. To verify the significant differences between the AA values of the proposed and existing methods, Wilcoxon tests (as described in Section 4.3) were applied, the results of which are shown in Table 6. It can be seen from Table 6 that significant differences were found in Bickerstaffe \& Zukerman's method [4] vs. the proposed method, Cao \& Zukerman's method [5] vs. the proposed method and Pang \& Lee's 
method [29] vs. the proposed method (with $\alpha=0.05$ ). A significant difference was also found in Goldberg \& Zhu's method vs. the proposed method [15] (with $\alpha=0.1$ ), but the significance of this difference is low, which is mainly due to the relatively small sample size of four data subsets used by Goldberg \& Zhu [15].

\section{Table 6}

Wilcoxon test results verifying the significant differences between the results of the existing and proposed methods $(K=20)$.

\begin{tabular}{|c|c|c|c|c|c|}
\hline Compared methods & $\mathrm{R}^{+}$ & $\mathrm{R}^{-}$ & Tie & Hypothesis & $p$-Value \\
\hline $\begin{array}{l}\text { Bickerstaffe \& Zukerman's } \\
\text { method [4] vs. the proposed } \\
\text { method }\end{array}$ & 0 & 36 & 0 & $\begin{array}{l}\text { Rejected for Bickerstaffe \& Zukerman at } \\
95 \%\end{array}$ & 0.012 \\
\hline $\begin{array}{l}\text { Cao \& Zukerman's method } \\
\text { [5] vs. the proposed method }\end{array}$ & 0 & 36 & 0 & Rejected for Cao \& Zukerman at $95 \%$ & 0.012 \\
\hline $\begin{array}{l}\text { Pang \& Lee's method [29] vs. } \\
\text { the proposed method }\end{array}$ & 0 & 36 & 0 & Rejected for Pang \& Lee at $95 \%$ & 0.012 \\
\hline $\begin{array}{l}\text { Goldberg \& Zhu's method } \\
\text { [15] vs. the proposed method }\end{array}$ & 0 & 10 & 0 & Rejected for Goldberg \& Zhu at $90 \%$ & 0.068 \\
\hline \multicolumn{6}{|c|}{$\begin{array}{l}\text { Notes: 1. 'Hypothesis' indicates that there is no significant difference between the results of the twc } \\
\text { methods. For example, the first Hypothesis can be read as "there is no significant difference } \\
\text { between the results obtained by Bickerstaffe \& Zukerman's method [4] and those obtained by the } \\
\text { proposed method". } \\
\text { 2: The } p \text {-value indicates the level of the significant difference between the results obtained by the two } \\
\text { methods. }\end{array}$} \\
\hline
\end{tabular}

\subsection{Comparing the improved and existing OVO strategies in multi-class sentiment} classification

In the existing studies, several OVO strategies have been proposed, such as the V-OVO [12], WV-OVO [17] and DRCW-OVO strategies [11]. To further illustrate the effectiveness of our improved OVO strategy, we compared the performance of the improved OVO strategy with those of the existing OVO strategies for multi-class sentiment classification. Following the framework shown in Fig. 1, each of the existing OVO strategies was used to identify the sentiment class of a test sample according to a score matrix $R=\left(r_{i i^{\prime}}\right)_{m \times m} .10$-fold cross validation was used to obtain the AA values of the different OVO strategies for identifying the sentiment classes of test samples in the data subsets. The obtained AA values are shown in Table 7. It can be seen from Table 7 that the AA values of the improved OVO and DRCWOVO strategies outperform each other depending on the data subsets and values of $K$ used, whereas the AA values of the improved OVO and DRCW-OVO strategies are clearly higher 
than those of the V-OVO and the WV-OVO strategies. Wilcoxon tests were used to verify the significant differences between the AA values of the improved and existing OVO strategies. Each data subset is associated with four AA values corresponding to the improved OVO and the DRCW-OVO strategies because different AA values were obtained for $K=1,5,10$ and 20 . Thus, to apply the Wilcoxon tests, each AA value of the V-OVO and the WV-OVO strategies was regarded as four similar values to guarantee an identical number of AA values for each OVO strategy. The Wilcoxon test results are shown in Table 8 . It can be seen from Table 8 that significant differences were found between the AA values of the improved and existing OVO strategies (with $\alpha=0.05$ ).

Table 7

AA values of various OVO strategies for identifying the sentiment classes of test samples in different data subsets.

\begin{tabular}{|c|c|c|c|c|c|c|c|c|c|c|}
\hline & \multicolumn{4}{|c|}{ Proposed OVO strategy } & \multicolumn{4}{|c|}{ DRCW-OVO strategy } & \multirow{2}{*}{$\begin{array}{l}\text { V-OVO } \\
\text { strategy }\end{array}$} & \multirow{2}{*}{$\begin{array}{c}\text { WV-OVO } \\
\text { strategy }\end{array}$} \\
\hline & $K=1$ & $K=5$ & $K=10$ & $K=20$ & $K=1$ & $K=5$ & $\begin{array}{c}K=1 \\
0\end{array}$ & $\begin{array}{c}K=2 \\
0\end{array}$ & & \\
\hline $\begin{array}{l}\text { Data subset } \\
1\end{array}$ & 74.02 & 72.83 & 72.83 & 72.83 & $\begin{array}{c}74.1 \\
5\end{array}$ & $\begin{array}{c}72.8 \\
3\end{array}$ & $\begin{array}{c}72.8 \\
3\end{array}$ & $\begin{array}{c}73.9 \\
1\end{array}$ & 72.70 & 72.49 \\
\hline $\begin{array}{l}\text { Data subset } \\
2\end{array}$ & 67.33 & 71.15 & 66.35 & 66.29 & $\begin{array}{c}67.4 \\
2\end{array}$ & $\begin{array}{c}70.1 \\
9\end{array}$ & $\begin{array}{c}65.3 \\
8\end{array}$ & $\begin{array}{c}66.0 \\
0\end{array}$ & 65.62 & 66.39 \\
\hline $\begin{array}{l}\text { Data subset } \\
3\end{array}$ & 86.31 & 82.44 & 86.57 & 86.59 & $\begin{array}{c}86.2 \\
6\end{array}$ & $\begin{array}{c}81.6 \\
8\end{array}$ & $\begin{array}{c}86.4 \\
8\end{array}$ & $\begin{array}{c}86.5 \\
9\end{array}$ & 85.81 & 86.22 \\
\hline $\begin{array}{l}\text { Data subset } \\
4\end{array}$ & 78.42 & 77.27 & 76.81 & 76.58 & $\begin{array}{c}78.4 \\
2\end{array}$ & $\begin{array}{c}77.4 \\
3\end{array}$ & $\begin{array}{c}76.7 \\
3\end{array}$ & $\begin{array}{c}76.2 \\
7\end{array}$ & 76.04 & 75.97 \\
\hline $\begin{array}{l}\text { Data subset } \\
5\end{array}$ & 80.49 & 80.15 & 80.04 & 80.15 & $\begin{array}{c}80.4 \\
9\end{array}$ & $\begin{array}{c}80.0 \\
4\end{array}$ & $\begin{array}{c}79.8 \\
2\end{array}$ & $\begin{array}{c}80.0 \\
4\end{array}$ & 79.38 & 79.71 \\
\hline $\begin{array}{l}\text { Data subset } \\
6\end{array}$ & 68.14 & 69.79 & 69.13 & 69.12 & $\begin{array}{c}68.0 \\
2\end{array}$ & $\begin{array}{c}69.6 \\
9\end{array}$ & $\begin{array}{c}69.0 \\
2\end{array}$ & $\begin{array}{c}68.7 \\
9\end{array}$ & 68.01 & 68.90 \\
\hline $\begin{array}{l}\text { Data subset } \\
7\end{array}$ & 80.43 & 80.32 & 80.37 & 80.32 & $\begin{array}{c}80.3 \\
7\end{array}$ & $\begin{array}{c}80.2 \\
6\end{array}$ & $\begin{array}{c}80.4 \\
9\end{array}$ & $\begin{array}{c}80.3 \\
2\end{array}$ & 80.37 & 80.43 \\
\hline $\begin{array}{l}\text { Data subset } \\
8\end{array}$ & 73.79 & 73.59 & 73.19 & 73.03 & $\begin{array}{c}73.7 \\
1\end{array}$ & $\begin{array}{c}73.5 \\
9\end{array}$ & $\begin{array}{c}73.0 \\
8\end{array}$ & $\begin{array}{c}73.0 \\
8\end{array}$ & 72.57 & 73.19 \\
\hline Average & 76.12 & 75.94 & 75.66 & 75.61 & $\begin{array}{c}76.1 \\
1\end{array}$ & $\begin{array}{c}75.7 \\
1\end{array}$ & $\begin{array}{c}75.4 \\
8\end{array}$ & $\begin{array}{c}75.6 \\
3\end{array}$ & 75.06 & 75.41 \\
\hline
\end{tabular}

Table 8

Wilcoxon test results for verifying the significant differences between the improved and existing OVO strategies.

$\mathrm{R}^{+} \quad \mathrm{R}^{-} \quad$ Hypothesis $\quad p$-Value




\begin{tabular}{|c|c|c|c|c|}
\hline $\begin{array}{l}\text { The improved OVO strategy vs. the V- } \\
\text { OVO strategy }\end{array}$ & 463 & 33 & Rejected for V-OVO at $95 \%$ & 0.000 \\
\hline $\begin{array}{l}\text { The improved OVO strategy vs. the } \\
\text { WV-OVO strategy }\end{array}$ & 390 & 75 & Rejected for WV-OVO at 95\% & 0.001 \\
\hline $\begin{array}{l}\text { The improved OVO strategy vs. the } \\
\text { DRCW-OVO strategy }\end{array}$ & 243.5 & 81.5 & Rejected for DRCW-OVO at $95 \%$ & 0.029 \\
\hline $\begin{array}{l}\text { Notes: 1. 'Hypothesis' indicates that the } \\
\text { improved and existing OVO strat } \\
\text { significant difference between the } \\
\text { 2: The } p \text {-value indicates the signif }\end{array}$ & improv & & $\begin{array}{l}\text { icant difference between the AA va } \\
\text { aple, the first Hypothesis states that " } \\
\text { strategy and the V-OVO strategy". } \\
\text { level between the AA values of the }\end{array}$ & $\begin{array}{l}\text { ues of the } \\
\text { there is no }\end{array}$ \\
\hline
\end{tabular}

\section{Conclusions}

We have presented a new method for multi-class sentiment classification using an improved OVO strategy and the SVM algorithm. First, we proposed an improved OVO strategy. Then, using this improved OVO strategy and the SVM algorithm, we proposed a method for multi-class sentiment classification. Several experimental studies were conducted to illustrate the effectiveness of the proposed method. The results of the experimental studies show that the performance of the proposed method is significantly better than the performance of the existing methods for multi-class sentiment classification. The major contributions of this paper are discussed as follows.

An improved OVO strategy is proposed wherein the relative competence weights of binary classifiers were determined according to the $K$ nearest neighbors and the class center of each class in the training sample sets. The improved OVO strategy overcomes the shortcoming of the existing OVO strategies, namely, that the competences of different binary classifiers are not considered or outliers would seriously affect the accuracy of the classification results. It is a valuable attempt to further improve the OVO strategies.

Second, the present paper presents a framework for multi-class sentiment classification using an improved OVO strategy and the SVM algorithm. This framework includes two stages: 1) selecting important text features using the IG algorithm and 2) identifying the sentiment classes of texts based on the improved OVO strategy and the SVM algorithm. Based on the framework, a detailed description of the proposed method was given. The framework lays a good foundation for conducting further studies on multi-class sentiment classification methods using improved OVO strategies and machine learning algorithms.

Third, experimental studies were conducted to verify the effectiveness of the proposed 
method. The results of the experimental studies show that the proposed method is robust with respect to different values of $K$ (the number of nearest neighbors selected from each sentiment class for determining the relative competence weight of each classifier). In addition, the proposed method showed significantly better performance than the existing methods on a multi-class sentiment classification task. The experimental results also show that the improved OVO strategy outperforms the existing OVO strategies on multi-class sentiment classification.

It is important to highlight that, because the proposed method is new and differs from the existing methods, it gives experts and decision analysts an additional option to consider. The proposed method is also important for the development and enrichment of theories and methods for multi-class sentiment classification.

With respect to future research, methods for supporting the decisions of governments, companies and consumers can be developed by integrating the proposed method with decision-analysis methods. In addition, although several methods for binary sentiment classification utilize deep learning [14, 33, 35, 38] can be found, a method for multi-class sentiment classification based on deep learning is lacking, which may represent a promising avenue for future research.

\section{Acknowledgements}

This work was partially supported by the National Science Foundation of China (Project Nos. 71371002 and 71571039), the Fundamental Research Funds for the Central Universities, China (Project Nos. N140607001), and the 111 Project (B16009).

\section{References}

1. R. Baeza-Yates, B. Ribeiro-Neto, Modern Information Retrieval, ACM Press, New York, 1999.

2. J.A. Balazs, J.D. Velásquez, Opinion mining and information fusion: A survey, Inf. Fusion 27 (2016) 95-110.

3. J.C. Bertot, P. T. Jaeger, D. Hansen, The impact of polices on government social media usage: Issues, challenges, and recommendations, Gov. Inform. Q. 29(1) (2012) 30-40. 
4. A. Bickerstaffe, I. Zukerman, A hierarchical classifier applied to multi-way sentiment detection, in: Proceedings of the 23rd International Conference on Computational Linguistics, 2010, pp. 62-70.

5. M.D. Cao, I. Zukerman, Experimental evaluation of a lexicon-and corpus-based ensemble for multi-way sentiment analysis, in: Proceedings of the Australasian Language Technology Association Workshop, 2012, pp. 52-60.

6. K. Chen, G. Kou, J. Shang, Y. Chen, Visualizing market structure through online product reviews: Integrate topic modeling, TOPSIS, and multi-dimensional scaling approaches, Electron. Commer. Res. Appl. 14(1) (2015) 58-74.

7. S. Demir, E.A. Sezer, H. Sever, Modifications for the cluster content discovery and the cluster label induction phases of the lingo algorithm, Int. J. Comput. Theory Eng. 6 (2) (2014) 86-90.

8. J. Demšar, Statistical comparisons of classifiers over multiple data sets, J. Mach. Learn. 7 (2006) 1-30.

9. J. Fürnkranz, E. Hüllermeier, S. Vanderlooy, Binary decomposition methods for multipartite ranking, Joint European Conference on Machine Learning and Knowledge Discovery in Databases, Springer Berlin Heidelberg, 2009, pp. 359-374.

10. M. Galar, A. Fernández, E. Barrenechea, H. Bustince, F. Herrera, Aggregation Schemes for binarization techniques. Methods' Description, Tech. Rep., Research Group on Soft Computing and Intelligent Information Systems (2011). http://sci2s.ugr.es/ovo-ova/ AggregationMethods Description.pdf $>$.

11. M. Galar, A. Fernández, E. Barrenechea, F. Herrera, DRCW-OVO: distance-based relative competence weighting combination for one-vs-one strategy in multi-class problems, Pattern Recogn. 48 (1) (2015) 28-42.

12. M. Galar, A. Fernández, E. Barrenechea, H. Bustince, F. Herrera, An overview of ensemble methods for binary classifiers in multi-class problems: Experimental study on one-vs-one and one-vs-all schemes, Pattern Recogn. 44 (8) (2011) 1761-1776.

13. S. García, J. Derrac, J. Cano, F. Herrera, Prototype selection for nearest neighbor classification: taxonomy and empirical study, IEEE Trans. Pattern Anal. Mach. Intell. 34 (3) (2012) 417-435.

14. X. Glorot, A. Bordes , Y. Bengio, Domain adaptation for large-scale sentiment 
classification: A deep learning approach, in Proceedings of the 28th International Conference on Machine Learning (ICML-11), 2011 pp. 513-520.

15. A.B. Goldberg, X. Zhu, Seeing stars when there aren't many stars: Graph-based semisupervised learning for sentiment categorization, In: Proceedings of the First Workshop on Graph Based Methods for Natural Language Processing, 2006, pp. 45-52.

16. M. Hall, E. Frank, G. Holmes, B. Pfahringer, P. Reutemann, I.H. Witten, The WEKA data mining software: An update, SIGKDD Explor. 11 (1) (2009) 10-18.

17. E. Hullermeier, S. Vanderlooy, Combining predictions in pairwise classification: An optimal adaptive voting strategy and its relation to weighted voting, Pattern Recogn. 43 (1) (2010) 128-142.

18. D. Kang, Y. Park, Review-based measurement of customer satisfaction in mobile service: Sentiment analysis and VIKOR approach, Expert Syst. Appl. 41 (4) (2014) 1041-1050.

19. F.H. Khan, U. Qamar, S. Bashir, eSAP: A decision support framework for enhanced sentiment analysis and polarity classification, Inf. Sci. 367 (2016) 862-873.

20. S. Knerr, L. Personnaz, G. Dreyfus, Single-layer learning revisited: A stepwise procedure for building and training a neural network, in: F. Fogelman Soulié, J. Hérault (Eds.), Neurocomputing: Algorithms, Architectures and Applications, vol. F68 of NATO ASI Series, Springer-Verlag, 1990, pp. 41-50.

21. Y.F. Lan, Y.K. Liu, G.J. Sun, Modeling fuzzy multi-period production planning and sourcing problem with credibility service levels, J. Comput. Appl. Math. 231 (1) (2009) 208-221.

22. A.J. Lee, F.C. Yang, C.H. Chen, C.S. Wang, C.Y. Sun, Mining perceptual maps from consumer reviews, Decis. Support Syst. 82 (2016) 12-25.

23. X. Li, H.S. Wong, S. Wu, A fuzzy minimax clustering model and its applications, Inf. Sci. 186 (1) (2012) 114-125.

24. X. Li, B. Liu, Chance measure for hybrid events with fuzziness and randomness, Soft Comput. 13 (2) (2009) 105-115.

25. Y.M. Li, H.M. Chen, J.H. Liou, L.F. Lin, Creating social intelligence for product portfolio design, Decis. Support Syst. 66 (2014) 123-134.

26. Z.Q. Liu, Y.K. Liu, Type-2 fuzzy variables and their arithmetic, Soft Comput. 14 (7) (2010) 729-747. 
27. Y.K. Liu, Z.Q. Liu, J. Gao, The modes of convergence in the approximation of fuzzy random optimization problems, Soft Comput. 13 (2) (2009) 117-125.

28. W. Medhat, A. Hassan, H. Korashy, Sentiment analysis algorithms and applications: A survey, Ain Shams Eng. J. 5 (4) (2014) 1093-1113.

29. B. Pang, L. Lee, Seeing stars: Exploiting class relationships for sentiment categorization with respect to rating scales, in: Proceedings of the 43rd Annual Meeting on Association for Computational Linguistics, 2005, pp. 115-124.

30. J.C. Platt, Fast Training of Support Vector Machines using Sequential Minimal Optimization, MIT Press, Cambridge, MA, USA, 1999.

31. R. Qin, Y.K. Liu, Z.Q. Liu, Methods of critical value reduction for type-2 fuzzy variables and their applications, J. Comput. Appl. Math. 235 (5) (2011) 1454-1481.

32. K. Ravi, V. Ravi, A survey on opinion mining and sentiment analysis: Tasks, approaches and applications, Knowl.-Based Syst. 89 (2015) 14-46.

33. Y. Ren, R. Wang, D. Ji, A topic-enhanced word embedding for Twitter sentiment classification, Inf. Sci. 369 (2016) 188-198.

34. J. Serrano-Guerrero, J.A. Olivas, F.P. Romero, E. Herrera-Viedma, Sentiment analysis: a review and comparative analysis of web services, Inf. Sci. 311 (2015) 18-38.

35. R. Socher, A. Perelygin, J. Y. Wu, et al., Recursive deep models for semantic compositionality over a sentiment treebank, in Proceedings of the conference on empirical methods in natural language processing (EMNLP). 2013 pp. 1631-1642.

36. P.J. Stone, D.C. Dunphy, M.S. Smith, D.M. Ogilvie, The general inquirer: A computer approach to content analysis, MIT Press, Cambridge, MA, 1966.

37. S. Tan, J. Zhang, An empirical study of sentiment analysis for Chinese documents, Expert Syst. Appl. 34 (4) (2008) 2622-2629.

38. D. Tang, B. Qin, T. Liu, Deep learning for sentiment analysis: successful approaches and future challenges, WIREs Data Min. Knowl. Disc., 5 (6) (2015) 292-303.

39. H. Tang, S. Tan, X. Cheng, A survey on sentiment detection of reviews, Expert Syst. Appl. 36 (7) (2009) 10760-10773.

40. M. Thelwall, K. Buckley, G. Paltoglou, D. Cai, A. Kappas, Sentiment strength detection in short informal text, J. Am. Soc. Ins. Sci. Technol. 61 (12) (2010) 2544-2558.

41. M. Thelwall, K. Buckley, G. Paltoglou, Sentiment strength detection for the social web, J. 
Am. Soc. Ins. Sci. Technol. 63(1) (2012), 163-173.

42. S. Wang, D. Li, X. Song, Y. Wei, H. Li, A feature selection method based on improved fisher's discriminant ratio for text sentiment classification, Expert Syst. Appl. 38 (7) (2011), 8696-8702.

43. F. Wilcoxon, Individual comparisons by ranking methods, Biometrics Bull. 1 (6) (1945) 80-83.

44. T. Wilson, J. Wiebe, R. Hwa, Recognizing strong and weak opinion clauses, Comput. Intell. 22 (2) (2006) 73-99.

45. X.F. Xu, J. Hao, Y.R. Deng, Y. Wang, Design optimization of resource combination for collaborative logistics network under uncertainty, Appl. Soft Comput. J. (2016) forthcoming http://dx.doi.org/10.1016/j.asoc.2016.07.036.

46. X. Yang, G. Yang, J. Wu, Integrating rich and heterogeneous information to design a ranking system for multiple products, Decis. Support Syst. 84 (2016) 117-133.

47. Y. Yang, J.O. Pedersen, A comparative study on feature selection in text categorization, in: Proceedings of the 14th International Conference on Machine Learning, 1997, pp. 412-420.

48. W. Zhang, H. Xu, W. Wan, Weakness finder: Find product weakness from Chinese reviews by using aspects based sentiment analysis, Expert Syst. Appl. 39 (11) (2012) 10283-10291. 